'Departamento de Medicina Interna Sur, Facultad de

Medicina, Universidad de Chile. Santiago, Chile.

2Departamento de

Endocrinología, Programa de Endocrinología Ginecológica, Escuela de Medicina, Pontificia Universidad Católica de Chile. Santiago, Chile.

Trabajo no recibió financiamiento. Los autores declaran no tener conflictos de interés.

Recibido el 9 de agosto de 2017, aceptado el 3 de octubre de

Correspondencia a:

Juan Enrique Blümel Orquídeas 1068. Dpto. 303.

Providencia 7510258

juan.blumel@redsalud.gov.cl jeblumel@vtr.net

\section{La terapia hormonal de la menopausia ¿disminuye el riesgo de enfermedades crónicas?}

\author{
JUAN ENRIQUE BLÜMEL ${ }^{1}$, EUGENIO ARTEAGA²
}

\section{Does menopause hormone therapy reduce the risk of chronic diseases?}

The hormonal deficit of post menopause is not only linked to the classic hot flashes, but also to a higher risk of chronic diseases. Menopausal hormone therapy (MHT) adequately treats climacteric symptoms and can prevent some chronic diseases such as osteoporosis. The Women's Health Initiative (WHI) study, which indicated risks of MHT in elderly postmenopausal women, caused a massive withdrawal of this therapy. But, in recent years the results of the WHI have been challenged by methodological problems and by several studies indicating that, if MHT is initiated early and the non-oral route is preferred, the risks could be minimized and it could improve not only the quality of life but also reduce the risk of chronic diseases. However, the US Preventive Services Task Force (USPSTF) recommends against the use of MHT for the prevention of chronic diseases, a position that has been challenged by publications of the North American Menopause Society and the International Menopause Society. This controversy persists so far. We report data that suggest a preventive role of MHT in perimenopausal women.

(Rev Med Chile 2018; 146: 1170-1174)

Key words: Chronic Diseases; Hormone Replacement Therapy; Postmenopause; Women's Health.
E: 1 climaterio no solo se asocia a un deterioro de la calidad de vida, sino que también a un aumento de las enfermedades crónicas ${ }^{1-3}$. Los estilos de vida sanos y la terapia hormonal de la menopausia (THM) son las principales intervenciones para contrarrestar los efectos del climaterio ${ }^{4}$. Sin embargo, el estudio Women's Health Initiative (WHI) que señaló que la THM tenía más riesgos que beneficios provocó prácticamente la desaparición del uso de esta terapia ${ }^{5,6}$. Pero, el no usar THM podría implicar no solo deterioro de la calidad de vida, sino que también se ha planteado que pudiéramos estar perdiendo una oportunidad de disminuir el riesgo de enfermedades crónicas en las mujeres postmenopáusicas ${ }^{7}$. Sin embargo, la US Preventive Services Task Force (USPSTF) recomienda contra el uso de la THM para la prevención primaria de enfermedades crónicas ${ }^{8}$. Estas recomendaciones fueron inmediatamente rebatidas por un grupo internacional de investigadores, entre los cuales estaba el presidente honorario de la Sociedad Norteamericana de Menopausia, que señalaron directamente que las recomendaciones de la USPSTF estaban equivocadas, ya que "los principales elementos de la evidencia en que se basa han sido mal interpretados o mal expresados, particularmente con respecto a la enfermedad coronaria y el cáncer de mama, para los cuales no hay evidencias estadísticamente significativas de daño" . En la presente revisión entregamos la visión de los autores en relación con la controversia planteada y los fundamentos en que se basan. 


\section{Enfermedad cardiovascular}

En la menopausia se produce aumento de la grasa abdominal e incremento del riesgo cardiovascular ${ }^{10}$. Esta asociación se ha concretado en un paradigma conocido como síndrome metabólico $^{11}$. El aumento de la grasa abdominal provoca un incremento en la síntesis de proteínas proinflamatorios y una disminución de las antiinflamatorias ${ }^{12}$, induciendo un estado inflamatorio crónico que aumenta el riesgo cardiovascular.

Pero no solo la obesidad aumenta el riesgo cardiovascular en la postmenopáusica, sino que también el déficit de estrógenos es determinante. Este esteroide disminuye la apoptosis celular y aumenta la resistencia del endotelio a la injuria, disminuye el paso de LDL plasmático hacia el espacio subedotelial, es antioxidante, disminuye la adhesión de monocitos y la respuesta quimiotáctica de los monocitos ${ }^{13,14}$. Por lo tanto, no es sorprendente que en las conejas castradas alimentadas con una dieta hipercolesterolémica se observe una mayor cantidad de lesiones ateroecleróticas que en las conejas con igual dieta, pero a las cuales no se le han extraído los ovarios ${ }^{15}$.

Hace dos décadas existía la idea que la THM disminuía el riesgo cardiovascular, pero el estudio WHI comunicó, en cambio, que aumentaba en $29 \%$ el riesgo coronario y en $41 \%$ los accidentes cerebrovasculares ${ }^{6}$. Estos resultados se atribuyeron al uso de terapia oral en mujeres mayores con lesiones ateroscleróticas, en las cuales el uso de la vía oral aumentaba el riesgo trombótico por hipercoagulabilidad e inestabilidad de las placas ateroscleróticas ${ }^{16}$. Esta hipótesis pareció confirmarse en otro estudio que encontró que la THM disminuía en $28 \%$ el riesgo coronario solo si se iniciaba en los primeros 10 años de postmenopausia $^{17}$. Posteriormente, el WHI mostró que la THM en menopáusicas recientes no aumentaba el riesgo coronario, mientras que las que iniciaban 20 años después de la menopausia lo incrementaba en $52 \%{ }^{18}$.

Otro estudio, ELITE, señaló que el tratamiento con estradiol oral se asociaba con una menor progresión de la aterosclerosis subclínica, evaluada por el grosos de la íntima y media de la arteria carótida, que el placebo, cuando el tratamiento se iniciaba dentro de los 6 años posteriores a la menopausia, pero no cuando se iniciaba $10 \mathrm{o}$ más años después de la menopausia ${ }^{19}$. De estas observaciones ha nacido el concepto de ventana de oportunidad: en mujeres menopáusicas recientes, con sistema cardiovascular sano, la THM sería cardioprotectora, a diferencias de las mujeres mayores, con arterias dañadas y con placas ateromatosas inestables, en quienes sería dañina.

Otros estudios han sugerido un rol cardioprotector de la THM en mujeres de mediana edad. Schierbeck en un estudio abierto, con distribución aleatoria, controlado, siguió durante 11 años a 1.006 mujeres tratadas con THM, encontrando que el riesgo cardiovascular era 0,48 (IC95\%, 0,26$0,87)^{20}$. Otro estudio, también observacional, que siguió a 489.105 mujeres usuarias de THM mostró que el riesgo de muerte coronaria se redujo en $54 \%$ y el de accidente cerebrovascular en 39\% en las usuarias de más de 10 años ${ }^{21}$. Por ello, no es posible, a la fecha, descartar un efecto cardioprotector de la THM cuando se indica antes de los 60 años.

\section{Osteoporosis}

En la menopausia, el déficit de estrógeno aumenta la resorción ósea mediada por osteoclastos, sin un aumento proporcional de la actividad osteoblástica, lo que lleva a un aumento de la pérdida ósea. La THM contrarresta los efectos óseos que provoca la menopausia ${ }^{22}$ y es el tratamiento que más directamente corrige la fisiopatología de esta enfermedad. Un metaanálisis muestra que, después de un año de uso, la densidad ósea aumenta $5,4 \%$ en columna lumbar, $3,0 \%$ en antebrazo y $2,5 \%$ en cuello femoral ${ }^{23}$. Otro metaanálisis mostró que, después de 5,6 años de THM combinada, la incidencia de fractura era 86 casos menos cada 1.000 mujeres tratadas comparada con las mujeres controles $^{24}$. Igualmente, el WHI, en pacientes no seleccionados por osteoporosis, mostró una disminución de $24 \%$ y $28 \%$ en el riesgo de fractura después de 13 años de seguimiento en las pacientes tratadas con THM combinada o con estrógenos solos, respectivamente ${ }^{18}$. Probablemente, la disminución de fracturas no solo se deba a su efecto antireabsortivo, sino que también a otros efectos positivos que tiene la THM. Un estudio que evaluó el efecto de la THM sobre las caídas, un fuerte predictor de fractura, señaló que las usuarias sufren menos caídas ${ }^{25}$. Resumiendo, los datos de disminución del riesgo de fractura en las usuarias de THM son bastante sólidos. 


\section{Cáncer}

El estudio WHI con estrógenos solos mostró una reducción de 23\% del riego relativo de cáncer de mamas en las usuarias de $\mathrm{THM}^{26}$. En cambio, en las mujeres con terapia combinada, el riesgo aumentaba $28 \%{ }^{27}$. Este último dato impactó fuertemente a la comunidad médica y al público en general. Sin embargo, los resultados de este último estudio han sido objetados porque el riesgo se calculó aplicando el modelo de riesgos proporcionales de Cox, sin cumplir con las condiciones que exige dicho modelo, lo cual invalida sus resultados. Al aplicar otro modelo matemático (Royston) a los mismos pacientes del WHI se observó que, después de cinco años, las usuarias de THM combinada presentaron cáncer de mama 1 día antes que las controles ${ }^{28}$. Igualmente, un análisis de Shapiro y cols., usando los criterios de causalidad de Bradford Hill, concluyó que el WHI no permite establecer que la THM aumente el riesgo de cáncer de mama ${ }^{29}$. Por lo tanto, la relación descrita entre cáncer de mama y THM permanece aún bastante confusa.

\section{Demencias}

La prevalencia de demencias en los mayores de 60 años fluctúa entre 5 y $9 \%{ }^{30}$, predominando en las mujeres. En lauchas transgénicas, que expresan características de la enfermedad de Alzheimer, los depósitos de $\beta$-amiloide son mayores en las hembras. En los humanos, la caída de los esteroides ováricos priva al cerebro de una serie de efectos anti-Alhzeimer, entre las cuales hay que mencionar una mejoría de la conectividad sináptica y de la neurotransmisión, menor muerte neuronal y menor acumulación de beta amiloides ${ }^{31}$. Existen receptores de estrógeno $\alpha$ y $\beta$ en el cerebro, siendo el más trascendente el $\alpha$, el cual desaparece en ausencia de estrógenos, pudiendo ser reinducidos solo si se reemplazan los estrógenos en forma relativamente precoz ${ }^{32}$.

Las mujeres ooforectomizadas antes de la menopausia muestran aumento del riesgo de demencia $^{33}$. Varios estudios han demostrado neuroprotección cuando la THM se inicia en la postmenopausia temprana ${ }^{34}$. Si la reposición hormonal se inicia tardíamente, en cambio, no se verifican los beneficios cognitivos. Esto es lo que sucedió con el estudio WHI, el uso de THM combinada en mujeres mayores de 65 años dobló el riesgo de demencia ${ }^{35}$. Contrariamente, en mujeres menores, la THM se asocia a una disminución de $65 \%$ del riesgo de enfermedad de Alzheimer ${ }^{36}$. Estas observaciones han llevado a plantear que existiría también una "ventana de oportunidad" para prevenir las demencias, pero, pasado los 65 años, el inicio de la terapia hormonal podría aumentar el riesgo ${ }^{37}$.

\section{Mortalidad}

Los positivos efectos de la THM en la calidad de vida y en el riesgo de enfermedades crónicas debieran traducirse en menor mortalidad. Un metaanálisis con 16.000 mujeres mostró que la mortalidad disminuía en $27 \%$ en las usuarias de $\mathrm{THM}^{38}$. Otro estudio, que siguió a casi medio millón de mujeres que usaron THM entre 1994 y 2009, señaló que el riesgo de morir de cualquier causa se redujo en $38 \%$ en las mujeres que usaron la terapia más de 10 años ${ }^{39}$. Contrariamente, otro metaanálisis, que incluyó a 38.908 mujeres, no mostró disminución de la mortalidad con $\mathrm{THM}^{40}$, sin embargo, este análisis incluyó 27.347 mujeres del WHI que tenían al inicio del estudio una edad promedio de 63,2 años, las cuales están fuera de la "ventana de oportunidad" antes enunciada. Pareciera que el uso precoz de THM podría asociarse a menor mortalidad.

\section{Conclusiones}

La menopausia deteriora la calidad de vida e incrementa el riesgo de enfermedades crónicas. La THM, aparte de su beneficio en control sintomático de la postmenopausia y en la calidad de vida, por sus múltiples acciones metabólicas, especialmente a nivel del sistema cardiovascular, nervioso central y esquelético, podría atenuar el riesgo de enfermedades crónicas. Aunque hoy las guías clínicas internacionales no recomiendan la THM para la prevención de las enfermedades crónica, nuevas evidencias en un futuro podrían hacer cambiar estos planteamientos. Por el momento, el juicio clínico bien informado, teniendo presente la relación de costo/beneficio de la reposición hormonal de la menopausia debería guiar la acción médica en este campo. 


\section{Referencias}

1. Blumel JE, Castelo-Branco C, Binfa L, Gramegna G, Tacla X, Aracena B, et al. Quality of life after the menopause: a population study. Maturitas 2000; 34: 17-23.

2. Khosla S. Pathogenesis of age-related bone loss in humans. J Gerontol A Biol Sci Med Sci 2013; 68: 1226-35.

3. Crandall CJ, Barrett-Connor E. Endogenous sex steroid levels and cardiovascular disease in relation to the menopause: a systematic review. Endocrinol Metab Clin North Am 2013; 42: 227-53.

4. Lobo RA, Davis SR, De Villiers TJ, Gompel A, Henderson VW, Hodis HN, et al. Prevention of diseases after menopause. Climacteric 2014; 17: 540-56.

5. Jewett PI, Gangnon RE, Trentham-Dietz A, Sprague BL. Trends of postmenopausal estrogen plus progestin prevalence in the United States between 1970 and 2010. Obstet Gynecol 2014; 124: 727-33.

6. Rossouw JE, Anderson GL, Prentice RL, LaCroix AZ, Kooperberg C, Stefanick ML, et al; Writing Group for the Women's Health Initiative Investigators. Risks and Benefits of Estrogen Plus Progestin in Healthy Postmenopausal Women: Principal Results From the Women's Health Initiative Randomized Controlled. JAMA 2002; 288: 321-33.

7. Langer RD. The evidence base for HRT: what can we believe? Climacteric 2017; 20: 91-6.

8. US Preventive Services Task Force. Hormone Therapy for the Primary Prevention of Chronic Conditions in Postmenopausal Women: US Preventive Services Task Force Recommendation Statement. JAMA 2017; 318: 2224-33.

9. Langer RD, Simon JA, Pines A, Lobo RA, Hodis HN, Pickar JH, et al. Menopausal hormone therapy for primary prevention: why the USPSTF is wrong. Climacteric 2017; 20: 402-13.

10. Davis SR, Castelo-Branco C, Chedraui P, Lumsden MA, Nappi RE, Shah D, et al. Understanding weight gain at menopause. Climacteric 2012; 15 (5): 419-29.

11. Grundy SM, Cleeman JI, Daniels SR, Donato KA, Eckel RH, Franklin BA, et al. Diagnosis and management of the metabolic syndrome: an American Heart Association/National Heart, Lung, and Blood Institute Scientific Statement. Circulation 2005; 112: 2735-52.

12. Nakamura K, Fuster JJ, Walsh K. Adipokines: a link between obesity and cardiovascular disease. J Cardiol 2014; 63: 250-9.

13. Arteaga E, Rojas A, Villaseca P, Bianchi M, Arteaga A, Durán D. In vitro effect of estradiol, progesterone, testosterone, and of combined estradiol/progestins on low density lipoprotein (LDL) oxidation in postmenopausal women. Menopause 1998; 5: 16-23.

14. Resanovic I, Rizzo M, Zafirovic S, Bjelogrlic P, Perovic M, Savic K, et al. Anti-atherogenic effects of $17 \beta$-estradiol. Horm Metab Res 2013; 45: 701-8.

15. Blümel JE, Castelo-Branco C, González P, Moyano C, Iturriaga M, Videla L, et al. Transdermal estrogens do not appear to modify the extent of lesional areas of aortic atherosclerosis in oophorectomized rabbits on a cholesterol-rich diet. Atherosclerosis 2000; 148: 303-8.

16. Rosano GM, Vitale C, Fini M. Hormone replacement therapy and cardioprotection: what is good and what is bad for the cardiovascular system?. Ann N Y Acad Sci 2006; 1092: 341-8.

17. Grodstein F, Manson JE, Stampfer MJ. Hormone therapy and coronary heart disease: the role of time since menopause and age at hormone initiation. J Womens Health (Larchmt) 2006; 15: 35-44.

18. Manson JE, Chlebowski RT, Stefanick ML, Aragaki AK, Rossouw JE, Prentice RL, et al. Menopausal hormone therapy and health outcomes during the intervention and extended post stopping phases of the Women's Health Initiative randomized trials. JAMA 2013; 310: 1353-68.

19. Hodis HN, Mack WJ, Henderson VW, Shoupe D, Budoff MJ, Hwang-Levine J, et al; ELITE Research Group. Vascular Effects of Early versus Late Postmenopausal Treatment with Estradiol. N Engl J Med 2016; 31; 374: 1221-31.

20. Schierbeck LL, Rejnmark L, Tofteng CL, Stilgren L, Eiken P, Mosekilde L, et al. Effect of hormone replacement therapy on cardiovascular events in recently postmenopausal women: randomized trial. BMJ 2012; 345: e6409.

21. Mikkola TS, Tuomikoski P, Lyytinen H, Korhonen P, Hoti F, Vattulainen P, et al. Estradiol-based postmenopausal hormone therapy and risk of cardiovascular and all-cause mortality. Menopause 2015; 22: 976-83.

22. Tella SH, Gallagher JC. Prevention and treatment of postmenopausal osteoporosis. J Steroid Biochem Mol Biol 2014; 142: 155-70.

23. Wells G, Tugwell P, Shea B, Guyatt G, Peterson J, Zytaruk N, et al; Osteoporosis Methodology Group and The Osteoporosis Research Advisory Group. Meta-analyses of therapies for postmenopausal osteoporosis. V. Meta-analysis of the efficacy of hormone replacement therapy in treating and preventing osteoporosis in postmenopausal women. Endocr Rev 2002; 23: 529-39.

24. Marjoribanks J, Farquhar C, Roberts H, Lethaby A. Long term hormone therapy for perimenopausal and postmenopausal women. Cochrane Database Syst Rev. 2012; 7: CD004143.

25. Bea JW, Zhao Q, Cauley JA, LaCroix AZ, Bassford T, 
Lewis CE, et al. Effect of hormone therapy on lean body mass, falls, and fractures: 6-year results from the Women's Health Initiative hormone trials. Menopause 2011; 18: 44-52.

26. LaCroix AZ, Chlebowski RT, Manson JE, Aragaki AK, Johnson KC, Martin L, et al; WHI Investigators. Health outcomes after stopping conjugated equine estrogens among postmenopausal women with prior hysterectomy: a randomized controlled trial. JAMA 2011; 305: 1305-14.

27. Manson JE, Chlebowski RT, Stefanick ML, Aragaki AK, Rossouw JE, Prentice RL, et al. Menopausal hormone therapy and health outcomes during the intervention and extended post stopping phases of the Women's Health Initiative randomized trials. JAMA 2013; 310: 1353-68.

28. Aedo S, Cavada G, Blümel JE, Chedraui P, Fica J, Barriga $\mathrm{P}$, et al. Women's Health Initiative estrogen plus progestin clinical trial: a study that does not allow establishing relevant clinical risks. Menopause 2015; 22: 1317-22.

29. Shapiro S, Farmer RD, Mueck AO, Seaman H, Stevenson JC. Does hormone replacement therapy cause breast cancer? An application of causal principles to three studies: part 2. The Women's Health Initiative: estrogen plus progestogen. J Fam Plann Reprod Health Care 2011; 37: 165-72.

30. Alzheimer's Disease International. World Alzheimer Report 2015. Disponible en: http://www.alz.co.uk/research/WorldAlzheimerReport2015-sheet.pdf. Accedida 28 Agosto 2018.

31. Rebekah SV, Pike CJ. Gender, sex steroid hormones, and Alzheimer's disease. Horm Behav 2013; 63: 301-7.

32. Hara Y, Waters EM, McEwen BS, Morrison JH, Estro- gen effects on cognitive and synaptic health over the lifecourse. Physiol Rev 2015; 95: 785-807.

33. Soni M, Hogervorst E. Premature ovarian insufficiency and neurological function. Minerva Endocrinol 2014; 39: 189-99.

34. Rocca WA, Grossardt BR, Shuster L. Oophorectomy, estrogen and dementia: A 2014 uptodate. Mol Cell Endocrinol 2014; 389: 7-12.

35. Shumaker SA, Legault C, Kuller L, Rapp SR, Thal L, Lane DS, et al; Women's Health Initiative Memory Study. Estrogen plus progestin and the incidence of dementia and mild cognitive impairment in postmenopausal women: The Women's Health Initiative Memory Study (WHIMS). JAMA 2003; 289: 2651-62.

36. Henderson VW, Benke KS, Green RC, Cupples LA, Farrer LA; MIRAGE Study Group. Postmenopausal hormone therapy and Alzheimer's disease risk: interaction with age. J Neurol Neurosurg Psychiatry 2005; 76: 103-5.

37. Jamshed N, Ozair FF, Aggarwal P, Ekka M. Alzheimer disease in post-menopausal women: intervene in the critical window period. J Mid-life Health 2014; 5: 38-40.

38. Salpeter SR, Cheng J, Thabane L, Buckley NS, Salpeter EE. Bayesian meta-analysis of hormone therapy and mortality in younger postmenopausal women. Am J Med 2009; 122: 1016-22.

39. Mikkola TS1, Tuomikoski P, Lyytinen H, Korhonen P, Hoti F, Vattulainen P, et al. Estradiol-based postmenopausal hormone therapy and risk of cardiovascular and all-cause mortality. Menopause 2015; 22: 976-83.

40. Yang D, Li J, Yuan Z, Liu X. Effect of hormone replacement therapy on cardiovascular outcomes: a meta-analysis of randomized controlled trials. PLoS One 2013; 8 (5): e62329. 